\title{
Current trends in biobanking for rare diseases: a review
}

Caroline E Graham'

Caron Molster'

Gareth S Baynam ${ }^{1-4}$

Kate Bushby ${ }^{5}$

Mats Hansson ${ }^{6}$

Anna Kole ${ }^{7}$

Marina Mora ${ }^{8}$

Lucia Monaco'

Matthew Bellgard ${ }^{10}$

David Carpentieri"

Manuel Posada'2

Olaf Riess ${ }^{13}$

Yaffa R Rubinstein ${ }^{14}$

Franz Schaefer ${ }^{15}$

Domenica Taruscio ${ }^{16}$

Sharon F Terry ${ }^{17}$

Kurt Zatloukal ${ }^{18}$

Bartha Knoppers ${ }^{19}$

Hanns Lochmüller ${ }^{5}$

Hugh JS Dawkins $1,10,20,21$

'Office of Population Health Genomics, Western Australian Department of Health, ${ }^{2}$ Genetic Services of Western Australia, Princess Margaret and King Edward Memorial Hospitals, ${ }^{3}$ School of Paediatrics and Child Health, University of Western Australia, ${ }^{4}$ Institute for Immunology and Infectious Diseases, Murdoch University, Perth, Australia; IInstitute of Genetic Medicine, MRC Centre for Neuromuscular Diseases, Newcastle University, Newcastle upon Tyne, UK; ${ }^{6}$ Centre Newcastle University, Newcastle upon Tyne, UK; ' $\mathrm{C}$ Centre for Research Ethics and Bioethics, Department of Public Health and Caring Sciences, Uppsala University, Uppsala, Sweden; ${ }^{7}$ European Organisation for Rare Diseases, Paris, France; ${ }^{8}$ Neuromuscular and Neuroimmunology Unit, C Besta Neurological Institute, ${ }^{9}$ Fondazione Telethon, Milan, Italy; ${ }^{10} \mathrm{C}$ nentre for Comparative Genomics, Murdoch University, Perth, Australia; "Phoenix Children's Hospital, University of Arizona, Mayo Medical School, Phoenix, AZ, USA; ${ }^{2}$ Institute of Rare Diseases Research, ISCIII, Instituto de Salud Carlos III, Madrid, Spain; ${ }^{13}$ Institute of Medical Genetics and Applied Genomics, Rare Disease Center Tuebingen, Tuebingen, Germany; ${ }^{14} \mathrm{Office}$ of Rare Diseases Research, National Cente for Advancing Translational Sciences, National Institutes of Health, Bethesda, MD, USA; ; ${ }^{\text {IS }}$ enter for Pediatrics and Adolescent Medicine, Division of Pediatric Nephrology, Heidelberg, Germany; ${ }^{16}$ National Center for Rare Diseases, Istituto Superiore di Sanità, Rome, Italy; ${ }^{17}$ Genetic Alliance, Washington, DC, USA: 18Institute of Pathology, Medicat Washington, DC, USA; Institute of Pathology, Medical University of Graz, Austria; "Centre of Genomics and Policy, Me Pelogy, "School or Australia, Perth, Australia

\section{Correspondence: Caroline E Graham}

Office of Population Health Genomics, Public Health and Clinical Services Division, Western Australian Department of Health, Level 3, C Block, 189 Royal St, East Perth, WA 6004, Australia $\mathrm{Tel}+61892226842$ Fax +61892226820

Email caroline.graham@health.wa.gov.au
This article was published in the following Dove Press journal:

Journal of Biorepository Science for Applied Medicine

21 November 2014

Number of times this article has been viewed

\begin{abstract}
Rare diseases (RD) refer to a collection of approximately 5,000-8,000 individual diseases that have a low prevalence and are often genetic in origin. While RD can manifest throughout life, they frequently affect children and newborns. Common characteristics include being severe, disabling, life-threatening, degenerative and affecting different organ systems. The burden of RD is often exacerbated by a lack of specific treatments. Whilst there is etiological heterogeneity, there is overlap in cellular and molecular pathways. Amongst specialists, there is legitimate hope that based on genetic knowledge and pathway definition, a new medical classification system, currently called "precision medicine", will be developed, which may change our view on how to apply shared therapeutic targets. Thus, collection of clinical and genetic data and biospecimens (in biobanks) will play an increasing role in diagnoses and development of therapies for RD. Biobanks are maintained collaboratively by researchers or their institutions, and involve a delicate balance between health policy objectives, academic research, public good outcomes, and community trust. Due to the nature of $\mathrm{RD}$, international cooperation is critical for sharing limited numbers of RD samples and achieving a critical mass. Here we review the current and future direction of RD biobanks and discuss research and development stemming from the use of biospecimens to improve management of RD.
\end{abstract}

Keywords: biobank, biospecimen, rare disease

\section{What are rare diseases?}

Rare diseases (RD), also called orphan diseases, refer to a collection of approximately 5,000-8,000 individual diseases that each have a prevalence of less than one in 2,000 and are often genetic in origin. ${ }^{1,2}$ The total number of individuals affected by RD worldwide is estimated at between 350 and 400 million. $^{3}$

While RD can manifest at any life stage, they often affect newborns and children. Common characteristics include being genetic, severe, disabling, mostly nonpreventable, life-threatening, degenerative, and having no effective treatments. ${ }^{1,4}$ RD also frequently affect different organ systems, requiring close interaction of different medical specializations. Whilst there is etiological heterogeneity, there are also thematic similarities across the RD natural history spectra that enable coordinated approaches. Those RD that are not solely genetic in nature can arise from exposure to drugs, chemicals, pollutants, infectious agents, and trauma; therefore, investigation of nongenetic risk factors will support primary prevention actions, eg, for rare congenital malformations. ${ }^{5,6}$ Within the broad group of RD, there exist distinct subgroups, which highlight further common pathologies. For example, they include, but are not limited to, subsets of metabolic diseases, rare cancers, autoimmune diseases, dysmorphic 
conditions, congenital malformations, and neuromuscular and neurodegenerative diseases.

The definition of RD promulgated by the European Commission is that they are of such low prevalence (less than one in 2,000 people) that special combined efforts are needed to address them. ${ }^{1,7}$ The US defines RD as diseases affecting a small percentage of the community, typically populations smaller than 200,000 individuals. ${ }^{8}$ Europe, Korea, and Singapore have a formalized definition that moves beyond being simply measured by prevalence. Recently, the Australian $\mathrm{RD}$ community proposed a definition for $\mathrm{RD}$ as being:

a life-threatening or chronically debilitating disease which is statistically rare (with an estimated prevalence of less than one in 2,000 or of similarly low prevalence) and has a high level of complexity such that special combined efforts are needed to address the disorder or condition. ${ }^{9}$

These definitions highlight the life-threatening nature and ongoing chronic burden of RD. Prevalence rates for a selection of RD are shown in Figure 1.

The rarity and diversity of RD pose specific challenges for health care provision and research and for the development and marketing of therapies. As a result, many patients with RD consult numerous doctors, do not receive a timely and accurate diagnosis, ${ }^{10}$ and few receive tailored treatments influencing survival and quality of life. Even today, only an additional six drugs on average receive "orphan drug" status worldwide per year. For these reasons, RD were made a priority area for action in the European Union Public Health Programme 2008-2013; multiple RD-focused projects have been funded under the 6 th and 7 th Framework Research
Programmes (FP), ${ }^{11}$ and more recently, Horizon 2020, which is the European Union's biggest research and innovation program with almost 80 million Euros of funding available, including a proportion specifically for RD initiatives. ${ }^{12}$ In recent years, the US government and the US FDA Office of Orphan Products Development have significantly increased the attention and support for RD and, as a result, the Office of Orphan Products Development has successfully enabled the development and marketing of more than 400 drugs and biological products for RD since $1983 .{ }^{13}$

The genetic diagnosis of a RD is often difficult due to clinical complexity, molecular interactions and lack of appropriate diagnostic tests. If available, the cost of validated tests may be prohibitive. Thus, they are usually only obtainable in highly specialized centers. Molecular characterization of the sample is critical for its use in research ${ }^{14}$ and a correct diagnosis, enabling patients to receive specific genetic counseling, potentially better care, and follow-up. Therefore, new diagnostic tools to enable research, development and marketing of therapies are needed.

\section{What are biobanks?}

The Organisation for Economic Co-operation and Development defines the term "biobank" as "a collection of biological material and the associated data and information stored in an organized system, for a population or a large subset of a population". ${ }^{15}$ These resources are maintained collaboratively by clinicians, researchers, and/or advocates, and often unite genetic information, biological samples, and patient experience. Biobanks involve a delicate balance between health policy objectives, academic research, public good

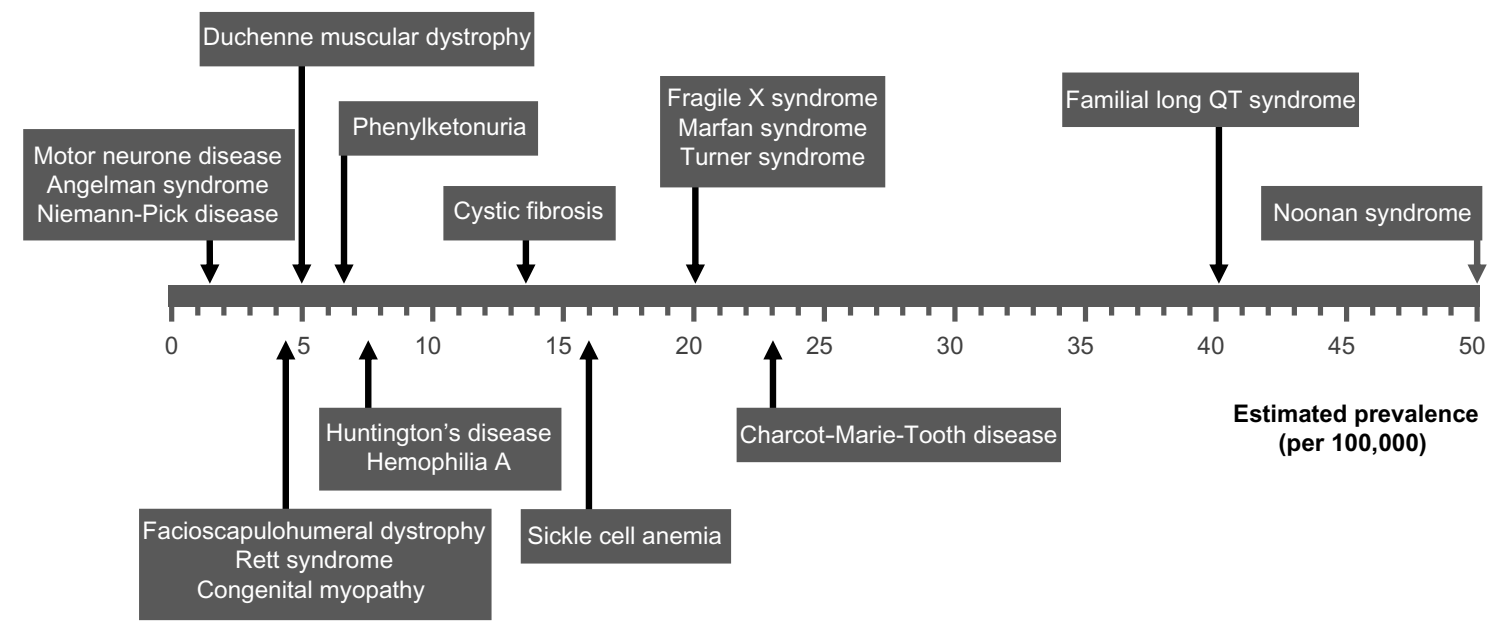

Figure I Some examples of rare disease prevalence rates. 
outcomes, community trust in the benefits of biobanks and protection of public interests such as privacy.

The nature of biological material stored by biobanks is varied. Biobanks can contain biospecimens from patients, family members and healthy individuals who can act as controls for specific study designs. Historically, biobanks can also store samples from animal studies; however, these biobanks are not covered under the scope of this review. New technologies provide important tools that allow researchers to collect and store many types of biological samples, such as blood, serum, DNA, RNA, spinal fluid, cells (including stem cells), and pathological tissue specimens. Cutting-edge research and translational medicine supported by biobanks and biospecimens are discussed in the section RD research and biobanking of this review.

Biobanks are an important resource that enables researchers, especially those in the RD field, access to limited and precious samples to study the underlying pathogenesis of a disease. ${ }^{16}$ New technologies, orphan drug legislation, networking and collaborations, RD community engagement, patient registries, objective assessments, bioinformatics support, policy support, ethical and legal frameworks and research and development are central to improving RD biobanks and, ultimately, RD management.

\section{Types of biobanks}

There are three main categories of biobanks, being diseasespecific, residual tissue or pathology, and longitudinal or population biobanks. They vary widely in size, ranging from small laboratory or single research team collections to larger networks of repositories, ${ }^{17}$ as follows:

- disease-specific biobanks contain biospecimens collected specific to a disease, such as the Australian Inherited Retinal Diseases Registry, ${ }^{18}$ and can form part of disease group biobanks such as the tumor repositories at the National Cancer Institute ${ }^{11,19}$

- residual tissue or pathology biobanks contain biological material removed during the course of diagnosis or treatment (eg, American Association of Tissue Banks, ${ }^{20}$ the BioVU project at Vanderbilt University, ${ }^{21}$ and the Genome Austria Tissue Bank ${ }^{22}$ )

- longitudinal and population biobanks are designed for relatively long-term linkage between biospecimens and health data, recruit participants from the general population and range from small town collections, such as the Busselton Health Study in Western Australia, ${ }^{23}$ through to nationwide studies, such as the UK Biobank, ${ }^{24}$ and more recently, networks that span regions of the globe.
Biobanks for RD can be designated for one specific disease, related diseases, or for all RD. Most of the RD biobanks are designated for a specific disease or class of diseases, eg, The Progeria Research Foundation Cell and Tissue Bank, National Institute of Child Health and Human Development Brain and Tissue Bank, Sterol and Isoprenoid Diseases Biospecimens/Biorepositories, National Mesothelioma Virtual Bank, Australian Inherited Retinal Diseases Register and DNA Bank, ${ }^{18}$ Munich Tissue Culture Collection, Myobank-AFM, and the DNA and cell bank at Genethon. On the other hand, the Coordination of Rare Diseases at Stanford, The Genetic Alliance Registry and BioBank (GARB), and National Rare Diseases Biobank, among others, are designated for all RD. Others, such as the National Disease Research Interchange store samples from common diseases as well as RD, in a well organized system that is labeled for easy identification and retrieval. There are more than 120 rare disease biobanks that are currently registered in the Orphanet database (http://www. orpha.net). The large size of many of these collections facilitates the analysis of rare genetic variants. ${ }^{25}$

\section{Biobank networks}

$\mathrm{RD}$ are defined by their low prevalence, which implies there are constraints and limitations for collecting a critical mass of cases for the same disease. To add to the complexity, RD often have a high variability in phenotype that necessitates large collections in order to provide enough statistical power to link genes and epigenetics or uncover other associations. For these reasons, collaborative efforts have been initiated by global networks to help identify and locate stores of biological material (DNA, tissues, cell cultures) pertaining to RD.

Disease-specific biobanks often come together to form networks. For example, under the leadership of the European Organisation for Rare Disease ${ }^{26}$ and funded by the European Union FP5, ${ }^{27}$ the EuroBioBank was the first network of RD biobanks to operate across Europe, with 16 biobanks from ten countries. ${ }^{14}$ Since 2012, Fondazione Telethon ${ }^{28}$ took charge of the EuroBioBank and its management, and also supports the Telethon Network of Genetic Biobanks (TNGB). ${ }^{29}$ It has been estimated that the EuroBioBank and TNGB store over half a million samples from over $500 \mathrm{RD}$, and that 13,000 samples are distributed worldwide per year. ${ }^{30}$

The National Institutes of Health (NIH) Rare Disease Hub (RD-HUB) is a centralized database of biorepositories for rare biospecimens established by the Office of Rare Diseases Research, National Center for Advancing Translational 
Sciences, NIH to aid researchers in locating RD biospecimens and facilitate the link between specimens and patient clinical data that resides in the Global Rare Disease Patient Register Data Repository (GRDR). ${ }^{31}$ GARB was developed to provide an infrastructure for advocacy organizations to establish and manage their biobanks and registries. It provides sophisticated technical solutions to advocacy organizations at a fraction of market cost, largely owing to cost sharing and the goodwill of their partnerships. ${ }^{32}$

The Biobanking and Biomolecular Resources Research Infrastructure (BBMRI) was funded to develop a coordinated, large-scale European infrastructure of biomedically relevant, quality assessed samples to enhance the prevention and treatment of diseases, including cancer. It will be implemented under the European Research Infrastructure Consortium (ERIC) legal entity by European member states, pulling together biobanks and biomolecular resources into a pan-European facility. ${ }^{33}$ BBMRI-ERIC will provide access to quality controlled collections of partner biobanks and biomolecular resources, their expertise, and services on a not-for-profit basis. BBMRI-ERIC foresees a central executive management office that coordinates the interaction of national nodes established in several member states. This will provide a common access portal to resources available for BBMRI-ERIC partners as well as appropriate facilities and expertise. Further examples of biobank networks can be found in Figure 2.

Biobank networks promote optimization and dissemination of quality banking practices, distribute quality material and associated data to scientific users, and disseminate biobank-pertinent knowledge and know-how to the scientific community. By improving accessibility of samples, biobank networks allow earlier and more accurate diagnoses as well as more effective treatments when available, and can improve medical and scientific collaboration in the field of RD.
RD-Connect is a global infrastructure project funded by the European Union FP7, although it is not in itself a biobank network. It is aimed at connecting databases, registries, biobanks, and clinical bioinformatics for RD research by developing robust mechanisms and standards for linking and exploiting data generated from RD research projects. ${ }^{30,34}$ To utilize the enormous power of the linked data and limited precious specimens, a suite of clinical bioinformatics tools, including data mining and knowledge discovery tools, are being developed within the RD-Connect framework. Further, major European research networks are underway, including NeurOmics ${ }^{35,36}$ which aims to expedite research on neurodegenerative and neuromuscular diseases and EURenOmics which aims to increase research on kidney diseases, and both directly interact with the RD-Connect network structure.

Linking biobanks and registries with other data sources ultimately enables clinicians and researchers to gain a better understanding of a disease. Providing secure access to data allows researchers in other institutions and those studying other RD to compare results and gain new insights. The Arizona Biospecimen Locator was built on an open source software framework that has served as a model for the Newborn Screening Translational Research Network (NBSTRN). The NBSTRN is an example of a successful network program which under the guidance of the American College of Medical Genetics and Genomics, refined a screening test for a rare immunodeficiency disorder. The underlying open source software program was further developed by a commercial information technology company and currently serves as a model for other virtual networks, like the Arizona Biospecimen Locator.

The rarity and diversity of RD and their associated biomaterials often require international collaboration and integration of data from different studies to achieve critical mass. ${ }^{14,37}$ The challenge is to ensure that this linking, or

\begin{tabular}{l|l} 
Network & Link \\
\hline Arizona biospecimen locator & https://abl.azdhs.gov/tissuelocator-web/browse.action \\
\hline Australasian biospecimen network (ABN) & www.abrn.net \\
\hline Biobanking and biomolecular resources research infrastructure (BBMRI-ERIC) & www.bbmri-eric.eu \\
\hline Cooperative human tissue network (CHTN) & www.chtn.nci.nih.gov \\
\hline EuroBioBank network & www.eurobiobank.org \\
\hline European rare tumors initiative & www.rarecancerseurope.org \\
\hline Genetic alliance registry and biobank (GARB) & www.biobank.org \\
\hline National rare disease biospecimen resource & www.ndriresource.org \\
\hline Newborn screening translation research network (NBSTRN) & www.nbstrn.org \\
\hline National rare diseases biobank (Instituto de Salud Carlos III) & https://spainrdr.isciii.es/en/Pages/Biobank.aspx \\
\hline NIH rare disease Hub (RD-HUB) & https://biospecimens.ordr.info.nih.gov \\
\hline New Zealand institute for rare disease research (NZIRDR) & http://www.nzirdr.org.nz/biobank \\
\hline Telethon network of genetic biobanks (TNGB) & www.telethon.it/en/scientists/biobanks \\
\hline Victorian cancer biobank & www.viccancerbiobank.org.au
\end{tabular}

Figure 2 Rare disease biobank networks.

Abbreviation: $\mathrm{NIH}$, National Institutes of Health. 
interoperability, is seamless and scalable. ${ }^{38}$ Compounding this challenge are the numerous legacy information systems currently in use that have not necessarily been designed with interoperability as a priority. One strategy to address this bottleneck is to build modular registry frameworks from which registries can be deployed with additional functionality and minimal longer-term software development effort. ${ }^{38,39}$

\section{RD research and biobanking}

Research provides a multitude of opportunities to further the scientific and clinical understanding of RD, as well as that of more common diseases. Whilst approximately 3,000 genes have been mapped to $\mathrm{RD}$, a further 3,000 RD are thought to be genetically determined, for which the genetic origin is currently unknown. ${ }^{40}$ An overview of the current environment highlights that $\mathrm{RD}$ research efforts tend to focus on diagnostics and therapeutics, spanning a range of approaches including translational and biomedical research, and drug development.

$\mathrm{RD}$ research has historically been highly fragmented by data type, research institution, and disease, and many still remain siloed. Until recently, biobanks designated to collect only samples from RD were few and far between. Those biobanks that did include samples of RD in their collection sometimes discarded these samples for lack of use. In other biobanks, with RD samples included, identification and availability of these samples was not adequate. Given that there are a limited number of biospecimens for most RDs and it may be difficult or impossible to obtain a new sample, these samples are extremely precious. Additionally, to achieve utility, the quality of the biomaterials and of the associated information is of primary importance. ${ }^{37}$ Siloed research biobanks held in laboratories with noninteroperable databases can make it almost impossible to connect genetic data with detailed clinical information or biospecimen availability. As the awareness of RD increases, more patient registries and biobanks are being established, allowing RD biospecimens to be more effectively identified, located and retrieved.

To accelerate progress in the field of $\mathrm{RD}$, major medical research funders have come together in a global effort aiming to foster collaboration in RD research on a worldwide basis. In fact, the European Commission has been supporting research into RD since the early 1990s under the European Union $\mathrm{FP}^{2}$ In particular, specific funding initiatives have been dedicated to biobanking activities, including the EuroBioBank network under FP5, the BBMRI-ERIC ${ }^{41,42}$ under FP7, and RD-Connect under FP7.
The International Rare Diseases Research Consortium (IRDiRC) was launched in 2011 and now has 34 members from across the world, including the key national funding agencies within the founding partners of the European Commission and the US NIH. Each of these funders has pledged to spend a minimum of US\$10 million on RD research over 5 years. The IRDiRC has set itself two aspirational objectives to achieve by the year 2020: to deliver 200 new therapies for RD and to develop the means to diagnose most RD. ${ }^{43}$ The IRDiRC policy and guidelines for research include the aim for RD patient registries and biobanks to be global in geographic scope and practice, interoperability and harmonization should be consistently pursued, linking and data transfer into existing platforms should be considered "best practice", and sharing and distributing of biomaterials among RD biobanks is highly encouraged. A specific working group on biobanks, within the IRDiRC's broader interdisciplinary scientific committee, has been established to help streamline access to harmonized data and samples. ${ }^{43}$

Clinical care, biomedical research practices, and the growth of biobanking have been greatly facilitated by advances in computing technology and bioinformatics. The use of such technologies is providing new opportunities to accumulate, share, mine, and integrate datasets for both clinical and research purposes, providing greater potential for growth in translational research. ${ }^{44}$ Networks such as eMERGE bring together biobanks with genomic data and access to clinical data from electronic medical records across multiple centres. ${ }^{45}$ The increased ability to link data is providing the mechanism for objective phenotyping and enabling translational research to become more efficient. Data from clinical registries and medical records are needed in order to assess outcomes in real-world clinical practice and also for evaluating the long-term effects of pharmaceutical treatment. Electronic health records are an important source of data that need to be associated with specimens, especially hospital integrated biobanks. These needs must be balanced against interests to protect privacy and appropriate levels of informed consent. ${ }^{46-48}$

\section{Clinical utility and benefits of RD biobanks}

Biobanks support the translation of laboratory research into clinical applications with the defined goal to develop diagnostic or therapeutic tools for disease. ${ }^{14,37}$ In $\mathrm{RD}$, where the source for this valuable biomaterial is limited and scattered over a large geographical area, biobanks are an important resource many times over. The benefits of biobanks vary depending on their purpose, which can be to: aid scientific 
discoveries and understanding of biology, causes of disease, genetic testing, replacement gene therapy, drug toxicity, efficacy, and treatment; identification of new genes and biomarkers; support clinical trials, education, and enable personalized medicine-targeting therapy.

Biospecimens held in biobanks have enabled researchers and clinicians to understand the mechanism and underlying cause of RD for gene discovery and for development of diagnostic and therapeutic biomarkers. Collections of DNA have been used to discover new genes and gene mutations, ${ }^{49-52}$ identify new diagnostic criteria, ${ }^{53}$ and genotype-phenotype correlations. ${ }^{54,55}$ On the other hand, sera and plasma have enabled the identification of new biomarkers ${ }^{56,57}$ and protein profiles to identify disease. ${ }^{58}$ Other types of biospecimens such as mRNA, cell lines, and tissues have assisted in collecting functional data to identify new pathways and new therapies to be applied to RD. ${ }^{59-63}$

Pharmaceutical companies utilize and benefit from the biobank networks, resulting in the successful development of clinical drugs for specific RD. ${ }^{64}$ More than 400 drugs and biological products for RD have been successfully developed and marketed since 1983, and the most recent drugs approved by the US FDA for RD from 2010 to $2013^{13}$ are listed in Figure 3. RD-HUB is one example of a central database for biorepositories/biospecimens of RD where pharmaceutical companies can locate and identify the specimens they need for their studies. In addition, RD-HUB facilitates linkage between patient clinical information and the corresponding biospecimens via the GRDR Global
Unique Identifier, which is one of the RD-HUB search fields. The GRDR Global Unique Identifier allows tracking of patient data over time and across studies, repositories, and countries. This tool is essential in the general process of developing drugs, eg, testing for drug efficacy, drug toxicity, and personalized medicine issues.

\section{Funding and sustainability}

Funding of RD biobanks is different from population and common disease biobanks which are usually supported by industry or public money. ${ }^{14}$ Many RD biobanks are supported by the proactive role of patients and patient organizations, short-term research grants, private donations, membership fees, or fees for service. Although more RD biobanks are supported by industry dollars each year, this poses a challenge. As there are a limited number of potential participants, if each company working on a disease creates a biobank, the collections will be very fragmented and burden a limited resource.

Securing long-term funding for developing and sustaining RD biobanks must follow fundamental business principles to achieve economies of scale, establish a compelling value proposition and understand the costs of doing business. ${ }^{65}$ The start-up infrastructure investment and operating cost requirements for even a modest biobank represent a significant commitment. ${ }^{66}$ There are several funding models for biobanks, which include private venture capital, government-funded, charitable not-for-profit and public-private partnerships. RD biobanks would benefit from a sustained

\begin{tabular}{|c|c|c|}
\hline Generic name & Orphan designation & Marketing approval \\
\hline Enalapril maleate & Treatment of hypertension in pediatric patients & $13 / 08 / 2013$ \\
\hline $\begin{array}{l}\text { Coagulation factor IX } \\
\text { (recombinant) }\end{array}$ & $\begin{array}{l}\text { Prophylactic use to prevent or reduce the frequency of bleeding episodes in } \\
\text { patients with hemophilia B (routine prophylaxis in patients where there is no } \\
\text { evidence or suspicion of bleeding) }\end{array}$ & $26 / 06 / 2013$ \\
\hline Afatinib & $\begin{array}{l}\text { Treatment of epidermal growth factor receptor (EGFR) mutation-positive non- } \\
\text { small cell lung cancer (NSCLC) }\end{array}$ & $12 / 07 / 2013$ \\
\hline Obinutuzumab & Treatment of chronic lymphocytic leukemia & $01 / 11 / 2013$ \\
\hline Dabrafenib & $\begin{array}{l}\text { Treatment of bioavailable inhibitor of B-raf (BRAF) protein V600 mutation positive } \\
\text { Stage IIB through IV melanoma }\end{array}$ & $29 / 05 / 2013$ \\
\hline Ibrutinib & Treatment of mantle cell lymphoma & $13 / 11 / 2013$ \\
\hline Ibrutinib & Treatment of chronic lymphocytic leukemia (CLL) & $12 / 02 / 2014$ \\
\hline Denosumab & Treatment of patients with giant cell tumor of bone & $13 / 06 / 2013$ \\
\hline $\begin{array}{l}\text { Anti-inhibitor coagulant } \\
\text { complex }\end{array}$ & $\begin{array}{l}\text { Routine prophylaxis to prevent or reduce the frequency of bleeding episodes in } \\
\text { hemophilia A and B patients with inhibitors }\end{array}$ & $16 / 12 / 2013$ \\
\hline Tasimelteon & Non-24-hour sleepwake disorder in blind individuals without light perception & $31 / 01 / 2014$ \\
\hline Trametinib and dabrafenib & Treatment of Stage IIb through IV melanoma & $10 / 01 / 2014$ \\
\hline Riociguat & Treatment of chronic thromboembolic pulmonary hypertension & $08 / 10 / 2013$ \\
\hline Riociguat & Treatment of pulmonary arterial hypertension & $08 / 10 / 2013$ \\
\hline Sorafenib & $\begin{array}{l}\text { Treatment of medullary thyroid cancer, anaplastic thyroid cancer, and recurrent or } \\
\text { metastatic follicular or papillary thyroid cancer }\end{array}$ & $22 / 11 / 2013$ \\
\hline
\end{tabular}

Figure 3 Drugs approved by the US Food and Drug Administration for rare diseases in 2010-2013. 
infrastructure. Establishing a biobank for any disease is extremely costly and time-consuming, and requires expert knowledge to maintain the physical infrastructure (including equipment) and data management of the inventory. The cost of the biobank is dependent on many factors, and not limited to the objectives of the biobank, size, location and type of samples. For biobanks specializing in RD, the cost is many times greater. The number of patients and samples collected are much smaller than for common diseases, such that, for commercial biobanks, the return over investment is not sufficient to make it an economical option. Unless there is a commitment to financially support biobanks specifically for $\mathrm{RD}$, these samples could be dispersed among samples of common diseases and be difficult to manage. RD-Connect is one example of attempts at infrastructure support in Europe. In the US, the NIH has two projects to connect many RD registries through shared infrastructure and GARB has been serving the goal of shared infrastructure since 2003 .

\section{Coding and ontologies}

Biobanks and collaborative research require sharing and/or integrating data coming from heterogeneous sources using a range of reference terminologies. Translation from one terminology into another requires semantic and syntactic interoperability. Until recently, there was no comprehensive nomenclature to classify and code RD. The World Health Organization's International Classification of Diseases Tenth Edition (ICD-10), now in worldwide clinical use, provides common, comparable diagnostic coding. However, it only provides specific coding for approximately 500 RD. ${ }^{37,67}$ This is likely to result in significant underreporting of $\mathrm{RD}$ and limits the ability of health systems to collect quality data for analyses.

Orphanet, an international consortium that provides a reference portal for $\mathrm{RD}$, has developed a comprehensive coding system for RD called Orphacodes, ${ }^{37,68}$ that is intended to be adopted in the ICD-11, due to be released in 2017. The Orphanet classification of RD has adopted a clinical approach by following the organization of medical specialties. It is multihierarchical, meaning that each entry is classified in one or more categories and in one or more sections of a single classification (multiple parentage). Orphacodes accommodate all known RD and, when linked to clinical and genetic data and disease classification, ensures comparability of data. ${ }^{14}$ Therefore, in the near future, there will be an internationally accepted, comprehensive data classification system that supports RD. Examples of biobank networks that have adopted Orphacodes include TNGB and the EuroBioBank.

\section{Return of incidental findings}

Findings from the use of biospecimens are generally aggregated and reported to the community in the form of newsletters, emails, articles, and posts on websites. Results specific to a participant that have potential health or clinical significance are termed "incidental findings" when they fall outside of the research objectives. ${ }^{17}$ Much debate has emerged over the obligations to return incidental findings to patients, and policies are rapidly being developed to cover actual and future obligations. ${ }^{69}$ In 2013, the American College of Medical Genetics and Genomics issued recommendations for reporting incidental findings from whole genome and whole exome sequencing used in clinical practice. If or how these guidelines should be implemented continues to be debated.

As yet, there are no overarching guidelines on the return of incidental findings to patients from biobanking research. Various approaches have been proposed, ranging from returning a specified set of results to actively consenting for non-return of incidental findings. Ultimately, an ethically defensible plan for return of incidental findings needs to be made, allowing for nuances between different jurisdictions. Accordingly, it has recently been argued that the time is not yet ripe for issuing recommendations. ${ }^{70}$

\section{Patient involvement and support}

The involvement of patients and patient organizations raises awareness, trust, and interest in biobanks, and has been demonstrated to be instrumental in gaining the critical mass of samples essential for research into RD that are very rare. ${ }^{29}$ Patients are not simply providers of biological material. ${ }^{14,37}$ Engagement of participants, families, and the community in biobanks is an essential component in decision-making. It is increasingly being recognized that patients are a valuable source of detailed information about their conditions and treatments and that this information can be integrated into research datasets.

As well as benefit sharing, some patients have advocated for shared participation. This creates a partnership between researchers and advocacy organizations, as is seen in a large number of collaborations led by advocacy organizations. ${ }^{55}$ Somewhat more complex systems also exist, such as the Platform for Engaging Everyone Responsibly ${ }^{71}$ and PatientsLikeMe. ${ }^{72}$ RD-HUB is a centralized database of biorepositories for rare biospecimens to aid researchers, ${ }^{73}$ and was created following a suggestion from a participant.

$\mathrm{RD}$ patient organizations recognize the importance of access to high-quality human biological materials as being a prerequisite for research. As such, many have started 
their own biobanks, recognizing that in combination with a registry, the biobank creates an excellent system for cohort development, a process that could otherwise be prohibitively time-consuming and difficult. As an example, in Europe, the EuroBiobank Network was established in 2001 by two patient organizations, ie, Association Française contre les Myopathies and the European Organisation for Rare Diseases. Soon after, the Genetic Alliance in the USA founded GARB. Both patient-centric initiatives have contributed heavily to the highest standards of sample collection, sample storage, sample sharing, and informed consent, which remain the gold standards today. Other recent examples of patient organizations directly establishing biobanks include the US Phelan-McDermid Foundation, ${ }^{74}$ the Italian Association for Alternating Hemiplegia, ${ }^{75}$ and the German Association for Families Affected by von Hippel-Lindau Syndrome. ${ }^{76}$

Still other patient groups have established special relationships with biobanks to increase patient participation. Agreements have been coordinated by the Italian National Alliance for Rare Disease patient organizations and the TNGB in Italy, where eight patient organizations have signed agreements with members of the Telethon Network. ${ }^{29}$ More specifically, the agreements help to raise awareness about the importance of participation in biobanking amongst support organizations and potential participants. This builds their capacity about issues in biobanking and prepares them to be empowered participants, allowing them to encourage their physicians to consider taking advantage of previously scheduled biopsies and extract and share biosamples with biobanks in the network. Above all, the agreement aims to ensure that samples are as widely available as possible for research purposes, while keeping patient interests in the core of activities and involving patients and their representatives in understanding the uses of their biospecimens.

Initiatives encouraging patient engagement will probably multiply in the near future, and for this reason the involvement of patient representatives in discussions on the future of biobanking for RD is key. This guarantees a patient-centric approach that ultimately leads to higher quality practice, better patient participation and retention, and meeting of all stakeholder expectations. The challenges of including participants remains such as obtaining consent, protecting participant privacy concerns, maintaining public trust, and shifting attitudes towards patients as more than research subjects. ${ }^{44}$ These sensitive ethical, legal, and social issues are particularly heightened in the field of RD. Many RD are pediatric, so custodians and researchers must consider storage of tissues of minors who one day will become adults. Many RD are life-threatening, so tissues are sometimes from deceased individuals. Regarding privacy, especially with genetic research, patients may be identified easily and misuse of information is possible.

Given the need to address issues of consent and improve current policies and practices, patient representatives are included in the governance of projects such as BBMRI and RD-Connect. In the context of the BBMRI preparatory phase, a patients' working group was established as part of the BBMRI stakeholders forum in recognition of the fact that engagement with patients and patient organizations as stakeholders in biobanking practices results in valuable partnerships and sources of input in biobanking research. ${ }^{30}$ Patient groups defined the basic principles for patient participation in the biobanking research infrastructure of BBMRI-ERIC. ${ }^{77}$

The RD-Connect project will enable scientists and clinicians worldwide to access a single centralized repository for omics data and for phenotypic and biomaterial information. The project serves as an opportunity for accelerating the rate at which patient clinical and genomic data is linked with biosamples, but also developing innovative ways in which patient participation in biobanking research systems can be improved. RD patient expectations regarding biobanking research systems will be explored through empirical research such as focus groups and deliberative debates.

Increasingly, recruitment for participation in registries and biobanks comes from the use of social media. Simple tools, such as Facebook, Twitter, and custom social networking sites, work to aggregate individuals for biobanking, and in some cases even facilitate the entire process through recruitment, sample collection, and reporting general results. ${ }^{32}$

\section{Governance and ethics}

Governance plays a crucial role in addressing the social and ethical challenges of biobanking. ${ }^{78}$ Proper governance is key to the success of biobanking initiatives, ie, it is pivotal to ensuring the security and protection of participants, maintaining public support and financing, and ensuring the availability of biospecimens for research. ${ }^{79-81}$ This is especially pertinent to $\mathrm{RD}$ biobanking efforts due to the rarity and diversity of biomaterials and the role played by patients and patient organizations. ${ }^{14}$ However, few efforts have defined what constitutes a proper governance framework in practice. ${ }^{78,82}$ Without a proper governance framework, RD biobanks may unduly add layers of oversight, exhibit "silo attitudes" that inhibit sample sharing, and lack the infrastructure required 
to meet the level of transnational collaboration and harmonization needed.

Currently, transnational and international collaborative research in $\mathrm{RD}$ is severely hampered by the lack of common quality standards, non-harmonized data management systems, and heterogeneity of ethical and legal requirements between countries. ${ }^{83}$ The current lack of standardization and interoperability also prevents the integration of and reuse of data generated in different research projects, resulting in significant duplication of effort and even limited reproducibility of research. ${ }^{83}$ The low prevalence of RD and high phenotype variability provide a strong incentive for collaboration across borders though, in turn, this creates a challenge for governance structures, eg, an ethics review system. A recent example of this is a clinical trial in juvenile dermatomyositis. ${ }^{84}$ In order to collect data for this study, comprising 130 patients from 30 different countries, participation of 103 clinical centers was needed. Due to variable implementation of a clinical trials directive in the 27 European Union member states, the process of ethical approval took 2 years. In some countries, it was sufficient to have ethical approval from one ethics committee, but in other countries approval from all regional/local ethics committees was needed. In all, 97\% of the ethical review committees gave their approval without requesting any change at all. There were three negative decisions, mainly related to the fact that the drug under study was not approved for use in children. Governance structures of this kind need to be changed. From the perspective of the patient, it is clear that spending 2 years on getting approval for uncontroversial research, which $97 \%$ of the ethics committees authorized without requiring any further information, is a waste of valuable resources and an unwanted delay of medical progress for RD patients. ${ }^{85}$

While RD biobanks have distinguishing features, they also share challenges similar to those of population biobanks. ${ }^{14}$ National regulation of biobanks has resulted in heterogeneous systems across countries, legal fragmentation, and a lack of standardized biospecimens and data, which hinders transnational sharing and international collaboration. ${ }^{79,86}$ Regulations and requirements for privacy are different in different countries, making material transfer between countries problematic. Issues such as harmonization of consent processes, confidentiality, and data protection in the sample collection and respect for patient autonomy are not completely addressed. Biobanks test such regulatory frameworks and raise a number of complex issues for society. Some biobank networks operate in a federated manner where groups of related biobanks from different regions or countries are under one umbrella with coordinated activities, whilst others can be centralized and operate under a tight system with less operating freedom for the individual biobank. The present challenge for a biobank and biobanking networks is to ensure compliance with all applicable norms, and governance mechanisms are needed that are global in nature. For example, most countries do not have a generic model of informed consent for biobanks and the terms of consent vary between studies. ${ }^{87}$

The EuroBiobank is one network facing the ethical, legal, and social issues related to biobanking activity across European countries. In the absence of a specific regulatory framework, using informal mechanisms such as professional guidance, establishment of biobank policies, and application of professional values and culture, the EuroBiobank has been able to progress and contribute to the development of regulations, as well as to establish a basis for international cooperation in the field of RD biobanking.

While presenting a generic framework for the governance of RD biobanks is beyond the scope of this paper, basic elements can be grouped into internal and external governance mechanisms. Internal governance mechanisms include the participation of patients and patient organizations through public engagement and incorporation of various committees, such as management and scientific and ethics committees that reflect various activities and expertise from clinicians, pathologists, and geneticists, to name a few. External mechanisms encompass legislation and regulations, international codes of conduct and guidelines, professional codes of conduct, funders' requirements, scientific peer review, ethics review, and public perception. The relationship between partners, rules for decisionmaking processes, ethical guidelines, activities, policies, and expected benefits should be defined in the biobank governance structure.

Some biobanks and biobanking networks have developed common best practice guidelines and charters that are freely available on the Internet, such as the EuroBioBank, TNGB, and the international Public Population Project in Genomics and Society. ${ }^{14,29,88}$ GARB has developed protocols and material transfer agreements for release of data and samples. Each member organization, with their respective biobank oversight committee, determines who will have access to their collection. Member organizations steward, govern, own, and manage their data and sample collections, determine what data and samples will be collected, and consent their participants. ${ }^{32}$

Within the TNGB, standardization has been mainly achieved through database management, use of a minimum 
common dataset, controlled terminologies, sample access policies, common informed consent forms, and quality control tools, ${ }^{29}$ although it needs to be qualified that the TNGB are biobanks held within a single country, ie, Italy. The BBMRI-ERIC aims to harmonize biobanks by keeping biological samples in the country of origin and following local laws and guidelines on their handling and use. ${ }^{63}$

Currently in Europe, there is a focus on major reform of regulation of data protection, including much debate over the consent process for biobanks and RD research. ${ }^{17,89}$ Specific informed consent enables the participant to choose the type of research performed on the biospecimens. It requires the participant to be informed of the research objectives, risks, benefits, and details of the procedures and have a level of understanding to make decisions. Specific consent places a greater need for ongoing communication with participants to keep them up to date on the research. However, specific consent is restrictive and does not allow for future unspecified research. In contrast, broad consent allows the biospecimen and data to be used for unspecified and unforeseen future research subject to ethics approval, and therefore is more flexible for administration of the biobank. The third type of consent is dynamic and, as the name suggests, is flexible and interactive. Participants are kept informed of the research projects using web-based technology. Which of these approaches should be applied to biobanking for RD is currently being debated.

It will be important to distinguish between the consent needed to contribute to a resource biobank meant to serve others, such as a longitudinal population study and diseasespecific biobanks that can involve the care of patients. There is a cost of specific consent in terms of decreased treatment opportunities where RD patients are particularly vulnerable to high numbers of dropouts or low responses to questions of renewed consent that should be taken into consideration..$^{90-92}$ Another important and difficult task for consideration is the need to reconsent patients at the time of legal age. As suggested above, children are often affected by RD, and reconsenting will become an increasing issue of concern. Finally, as the need for stem cell banks increases, the need for appropriate ethical and scientific standards cannot be ignored.

\section{Next steps in biobanking for RD}

Further efforts are required to bring RD biobanks to the attention of scientists, clinicians, and patients, and to closely link information received from specialized diagnostic centers and disease experts for qualitative and quantitative improvements. ${ }^{14}$ Clinical staff should have a clear understanding of the added value of participating in the biobank network and be encouraged to collect samples and update relevant databases. Their efforts are a generous contribution to global health research, but also a direct way of improving their own scientific and medical activities. ${ }^{93}$ An accreditation and evaluation system should be established to recognize biobanks that provide high-quality samples, and reward and acknowledge scientists who establish and maintain highquality biobanks.

The legal and regulatory frameworks that apply to the biobanking area need to be synchronized to unify and simplify the practice of biobanking across multiple institutions and different countries. The current variability in biospecimen collection procedures will potentially result in varying biosample properties that may undermine future investigation. Mandatory use of standard operating procedures should be introduced to ensure data harmonization between biobanks. In addition, interoperability and harmonization between RD patient registries and RD biobanks is critical. This is in combination with procedures that are time-efficient and adapted to the clinical workflow that will promote clinical engagement and enhance diagnostic and therapeutic development for RD. Further, management of incidental findings is important to avoid dissemination of laboratory results from nonaccredited research or commercially oriented laboratories and maintain patient-centric approaches to RD management. RD-Connect has developed a charter to provide a common overview and foundational framework for the practice of data sharing, and to frame a minimal list of the terms needed to achieve an equitable and ethically grounded data sharing agreement through engagement and consensus with patients, clinicians, institutions, and government agencies. ${ }^{94}$

There is also great demand to add more extensive clinical data to RD biospecimens already collected and stored for research, and to link this to genetic data from whole exome or whole genome sequencing methods. This will enable researchers to better understand cellular interactions in response to therapeutic compound screening. Thus, biobanks will become a true resource of material and information to enhance RD translational research.

\section{Acknowledgments}

The authors acknowledge the financial support of the European Union Seventh Framework Programme (FP7/2007-2013) under grant agreement numbers 305444 (RD-Connect), 305608 (EURenOmics), and 305121 (NeurOmics); TREAT-NMD operating grants, FP6 LSHM-CT-2006-036825, 20123307 UNEW_FY2013; Telethon grant GTB12001 to TNGB, and AFM 16104; EUROPLAN 2012-2015, coordinated by 
Italian National Institute of Health-Italian National Centre for Rare Diseases, included in the EUCERD Joint Action (grant number 201122 01, cofunded by the European Union Commission (DG-SANCO); Office of Rare Diseases Research at National Center for Advancing Translational Sciences/ National Institutes of Health; European Organisation for Rare Diseases; Biobanking and Biomolecular Resources Research Infrastructure (BBMRI-ERIC); ISBER/ESBB Rare Diseases Working Group; and Public Population Project in Genomics and Society (P3G).

\section{Disclosure}

The authors report no conflicts of interest in this work.

\section{References}

1. European Organisation for Rare Diseases. Rare Diseases: understanding this public health priority, 2005. Available from: http://www.eurordis org/IMG/pdf/princeps_document-EN.pdf. Accessed May 7, 2014.

2. Ayme S, Rodwell C. Report on the state of the art of rare disease activities in Europe of the European Union Committee of Experts on Rare Diseases - Part I: Overview of rare disease activities in Europe and key developments in 2010. European Union Committee of Experts on Rare Diseases; 2013. Available from: http://www.eucerd.eu/?post_ type $=$ document $\& p=1378$. Accessed May 5, 2014.

3. The Global Genes Project. Rare facts and statistics. Statistics and figures on prevalence of rare and genetic diseases, 2014. Available from: http:// globalgenes.org/rarefacts. Accessed February 5, 2014.

4. Jaffe A, Zurynski Y, Beville L, Elliott E. Call for a national plan for rare diseases. J Paediatr Child Health. 2009;46:2-4.

5. European Surveillance of Congentical Anomalies. EUROCAT special report: congenital anomalies are a major group of mainly rare diseases Available from: http://www.eurocat-network.eu/content/EUROCATEUROPLAN-Primary-Preventions-Reccomendations.pdf. Accessed May 7, 2014.

6. Taruscio D, Arriola L, Baldi F, et al. European recommendations for primary prevention of congenital anomalies: a joined effort of EUROCAT and EUROPLAN projects to facilitate inclusion of this topic in the national rare disease plans. Public Health Genomics. 2014;17:115-123.

7. Orphanet. About rare diseases, 2012. Available from: http://www. orpha.net/consor/cgi-bin/Education_AboutRareDiseases.php?lng=EN. Accessed March 14, 2014

8. United States of America, Rare Diseases Orphan Product Development Act of 2002, HR 4014 (2002). Available from: http://www.govtrack.us/ congress/bill.xpd?bill=h107-4014. Accessed May 7, 2014.

9. Dawkins HJ, Molster CM, Youngs LM, O'Leary PC. Awakening Australia to rare diseases: symposium report and preliminary outcomes. Orphanet J Rare Dis. 2011;6:57.

10. Hilbert JE, Ashizawa T, Day JW, et al. Diagnostic odyssey of patients with myotonic dystrophy. J Neurol. 2013;260:2497-2504.

11. Publications Office of the European Union. Rare diseases - how Europe is meeting the challenges, 2013. Available from: http://ec.europa.eu/ research/health/pdf/rare-diseases-how-europe-meeting-challenges_ en.pdf. Accessed May 7, 2014.

12. European Commission. Horizon 2020, the EU Framework Programme for Research and Innovation, 2014. Available from: http://ec.europa.eu/ programmes/horizon2020/en. Accessed February 7, 2014.

13. US Food and Drug Administration. Available from: http://www.fda.gov/ default.htm. Accessed February 18, 2014.

14. Lochmuller H, Schneiderat P. Biobanking in rare disorders. Adv Exp Med Biol. 2010;686:105-113.
15. Organisation for Economic Co-Operation and Development. Glossary of Statistical Terms - Biobank, 2006; Available from: http://stats.oecd. org/glossary/detail.asp?ID=7220. Accessed February 7, 2014.

16. Asslaber M, Zatloukal K. Biobanks: transnational, European and global networks. Brief Funct Genomic Proteomic. 2007;6:193-201.

17. Knoppers BM, Zawati MH, Kirby ES. Sampling populations of humans across the world: ELSI issues. Annu Rev Genomics Hum Genet. 2012;13:395-413.

18. De Roach JN, McLaren TL, Paterson RL, et al. Establishment and evolution of the Australian Inherited Retinal Disease Register and DNA Bank. Clin Experiment Ophthalmol. 2013;41:476-483.

19. National Cancer Institute. Available from: http://www.cancer.org Accessed February 11, 2014.

20. American Association of Tissue Banks. Available from: http://www. aatb.org. Accessed February 11, 2014.

21. Vandervilt University Medical Centre Office of Research. BioVU project, 2014. Available from: http://www.vanderbilt.edu/oor/cores/ biovu-vanderbilt-dna-databank. Accessed February 11, 2014.

22. Asslaber M, Abuja PM, Stark K, et al. The Genome Austria Tissue Bank (GATiB). Pathobiology. 2007;74:251-258.

23. Busselton Health Study. Available from: http://www.busselton healthstudy.com. Accessed February 11, 2014.

24. UK Biobank. Available from: http://www.ukbiobank.ac.uk/. Accessed February 11, 2014.

25. Wolf SM, Crock BN, Van Ness B, et al. Managing incidental findings and research results in genomic research involving biobanks and archived data sets. Genet Med. 2012;14:361-384.

26. Rare Diseases Europe. Available from: http://www.eurordis.org. Accessed February 11, 2014.

27. European Commission. Public Health Programme 2008-2013. Available from: http://ec.europa.eu/health/programme/policy/2008 2013/. Accessed January 7, 2013.

28. Fondazione Telethon. Available from: https://www.telethon.it/en. Accessed February 11, 2014.

29. Filocamo M, Baldo C, Goldwurm S, et al. Telethon Network of Genetic Biobanks: a key service for diagnosis and research on rare diseases. Orphanet J Rare Dis. 2013;8:129.

30. Monaco L, Crimi M. The challenge for a European network of biobanks for rare diseases taken up by RD-Connect. Pathobiology. 2014. In press, 2014.

31. National Center for Advancing Translational Sciences. Rare disease human biospecimens/biorepositories, 2014. Available from: http:// biospecimens.ordr.info.nih.gov/. Accessed February 11, 2014.

32. Terry SF, Horn EJ, Scott J, Terry PF. Genetic Alliance Registry and BioBank: a novel disease advocacy-driven research solution. Per Med. 2011;8:207-213.

33. Biobanking and Biomolecular Resources Research InfrastructureEuropean Research Infrastructure Consortium. Available from: http:// bbmri-eric.eu/. Accessed February 14, 2014.

34. RD-Connect. Available from: http://www.rd-connect.eu. Accessed February 11, 2014.

35. NeurOmics [webpage on the Internet]. Neuromics; 2014. Available from: http://www.rd-neuromics.eu/. Accessed 11 Feb 2014.

36. EuRenOmics [webpage on the Internet]. EURenOmics; 2014. Available from: http://www.eurenomics.com/. Accessed 11 Feb 2014.

37. Lochmuller H, Ayme S, Pampinella F, et al. The role of biobanking in rare diseases: European Consensus Expert Group report. Biopreserv Biobank. 2009;7:155-156.

38. Bellgard M, Beroud C, Parkinson K, et al. Dispelling myths about rare disease registry system development. Source Code Biol Med. 2013;8:21.

39. Bellgard MI, Macgregor A, Janon F, et al. A modular approach to disease registry design: successful adoption of an internet-based rare disease registry. Hum Mutat. 2012;33:E2356-E2366.

40. Government of Western Australia Department of Health, Office of Population Health Genomics. A scoping paper for the need for a national rare diseases plan for Australia. Available from: http://www.genomics. health.wa.gov.au/publications/docs/Scoping_Paper_National_RD_ Plan_September_2013.pdf. Accessed May 7, 2014. 
41. Wichmann HE, Kuhn KA, Waldenberger M, et al. Comprehensive catalog of European biobanks. Nat Biotechnol. 2011;29: 795-797.

42. Yuille M, van Ommen GJ, Brechot C, et al. Biobanking for Europe. Brief Bioinform. 2008;9:14-24.

43. International Rare Disease Research Consortium. Policies and guidelines, 2013. Available from: http://www.irdirc.org. Accessed February 12, 2014.

44. Kaye J, Curren L, Anderson N, et al. From patients to partners: participant-centric initiatives in biomedical research. Nat Rev Genet. 2012;13:371-376.

45. eMERGE [web page]. 2014; Available from: http://emerge. mc.vanderbilt.edu/. Accessed June 16, 2014.

46. Eichler HG, Pignatti F, Flamion B, Leufkens H, Breckenridge A. Balancing early market access to new drugs with the need for benefit/risk data: a mounting dilemma. Nat Rev Drug Discov. 2008;7:818-826.

47. Hansson M. Where should we draw the line between quality of care and other ethical concerns related to medical registries and biobanks? Theor Med Bioeth. 2012;33:313-323.

48. Hansson MG. Taking the patient's side: the ethics of pharmacogenetics. Per Med. 2010;7:75-85.

49. Magri F, Del Bo R, D’Angelo MG, et al. Clinical and molecular characterization of a cohort of patients with novel nucleotide alterations of the dystrophin gene detected by direct sequencing. BMC Med Genet. 2011;12:37.

50. Ronchi D, Garone C, Bordoni A, et al. Next-generation sequencing reveals DGUOK mutations in adult patients with mitochondrial DNA multiple deletions. Brain. 2012;135:3404-3415.

51. Shirley MD, Tang H, Gallione CJ, et al. Sturge-Weber syndrome and port-wine stains caused by somatic mutation in GNAQ. $N$ Engl J Med. 2013;368:1971-1979.

52. Del Bo R, Tiloca C, Pensato V, et al. Novel optineurin mutations in patients with familial and sporadic amyotrophic lateral sclerosis. J Neurol Neurosurg Psychiatry. 2011;82:1239-1243.

53. Scionti I, Greco F, Ricci G, et al. Large-scale population analysis challenges the current criteria for the molecular diagnosis of fascioscapulohumeral muscular dystrophy. Am J Hum Genet. 2012;90: 628-635.

54. Anichini A, Fanin M, Vianey-Saban C, et al. Genotype-phenotype correlations in a large series of patients with muscle type CPT II deficiency. Neurol Res. 2011;33:24-32.

55. Terry SF, Terry PF, Rauen KA, Uitto J, Bercovitch LG. Advocacy groups as research organizations: the PXE International example. Nat Rev Genet. 2007;8:157-164.

56. Quero C, Colome N, Rodriguez C, et al. Proteomics of toxic oil syndrome in humans: phenotype distribution in a population of patients. Chem Biol Interact. 2011;192:129-135.

57. Ramirez RL, Qian J, Santambrogio P, Levi S, Koeppen AH. Relation of cytosolic iron excess to cardiomyopathy of Friedreich's ataxia. Am J Cardiol. 2012;110:1820-1827.

58. Lisitsa A, Moshkovskii S, Chernobrovkin A, Ponomarenko E, Archakov A. Profiling proteoforms: promising follow-up of proteomics for biomarker discovery. Expert Rev Proteomics. 2014;11:121-129.

59. Abujarour R, Bennett M, Valamehr B, et al. Myogenic differentiation of muscular dystrophy-specific induced pluripotent stem cells for use in drug discovery. Stem Cells Transl Med. 2014;3:149-160.

60. Sproul AA, Vensand LB, Dusenberry CR, et al. Generation of iPSC lines from archived non-cryoprotected biobanked dura mater. Acta Neuropathol Commun. 2014;2:4.

61. Barnes EA, Kenerson HL, Jiang X, Yeung RS. Tuberin regulates E-cadherin localization: implications in epithelial-mesenchymal transition. Am J Pathol. 2010;177:1765-1778.

62. Fausther M, Pelletier J, Ribeiro CM, Sevigny J, Picher M. Cystic fibrosis remodels the regulation of purinergic signaling by NTPDase1 (CD39) and NTPDase3. Am J Physiol Lung Cell Mol Physiol. 2010;298:L804-L818.
63. Keogh B. European Biobanks forge cross-border ties. J Natl Cancer Inst. 2011;103:1429-1431.

64. Abstracts of the 6th European Conference on Rare Diseases and Orphan Products. Brussels, Belgium; May 23-25, 2012. Orphanet J Rare Dis. 2012;7 Suppl 2:A1-A41.

65. Vaught J, Rogers J, Carolin T, Compton C. Biobankonomics: developing a sustainable business model approach for the formation of a human tissue biobank. J Natl Cancer Inst Monogr. 2011;2011:24-31.

66. Baird PM, Frome RJ. Large-scale repository design. Cell Preserv Technol. 2005;3:256-266.

67. Šimerka P, Eur-LEX, Access. Council Recommendation of 8 June 2009 on an action in the field of rare diseases. Official Journal of the European Union; 2009. 2009/C 151/02. Available from: http://eur-lex. europa.eu/LexUriServ/LexUriServ.do?uri=OJ:C:2009:151:0007:0010: EN:PDF. Accessed May 7, 2014.

68. Rath A, Olry A, Dhombres F, Brandt MM, Urbero B, Ayme S. Representation of rare diseases in health information systems: the Orphanet approach to serve a wide range of end users. Hum Mutat. 2012;33:803-808

69. Knoppers BM, Deschenes M, Zawati MH, Tasse AM. Population studies: return of research results and incidental findings policy statement. Eur J Hum Genet. 2013;21:245-247.

70. Viberg J, Hansson MG, Langenskiold S, Segerdahl P. Incidental findings: the time is not yet ripe for a policy for biobanks. Eur J Hum Genet. 2014;22:437-441.

71. Terry SF, Shelton R, Biggers G, Baker D, Edwards K. The haystack is made of needles. Genet Test Mol Biomarkers. 2013;17:175-177.

72. Wicks P, Vaughan T, Heywood J. Subjects no more: what happens when trial participants realize they hold the power? BMJ. 2014;348:368.

73. Rubinstein YR, Groft SC, Bartek R, et al. Creating a global rare disease patient registry linked to a rare diseases biorepository database: Rare Disease-HUB (RD-HUB). Contemp Clin Trials. 2010;31: 394-404.

74. Phelan-McDermid Syndrome Foundation. Available from: http://22q13. org/j15/. Accessed February 12, 2014.

75. Biobank and Clinical Registry for Alternating Hemiplegia. Available from: http://www.ibahc.org. Accessed February 12, 2014.

76. German Association for Families Affected by von Hippel-Lindau Syndrome Available from: http://www.hippel-lindau.de/biomaterialbank. html. Accessed February 12, 2014.

77. Griffith M, Mitchell D. Consultation document: basic principles for patient participation in BBMRI, 2010. Available from: http://bbmri. eu/documents/15600/19102/Consultation_Document_010810.pdf/ b7fcbd43-9ae6-4974-b066-6a573425b882. Accessed February 11, 2014.

78. O’Doherty KC, Hawkins A. Structuring public engagement for effective input in policy development on human tissue biobanking. Public Health Genomics. 2010;13:197-206.

79. Publications Office of the European Union. Biobanks for Europe: a challenge for governance. Available from: http://ec.europa.eu/ research/science-society/document_library/pdf_06/biobanks-foreurope_en.pdf. Accessed May 7, 2014.

80. Staunton C, Moodley K. Challenges in biobank governance in SubSaharan Africa. BMC Med Ethics. 2013;14:35.

81. Gottweis H, Lauss G. Biobank governance: heterogeneous modes of ordering and democratization. J Community Genet. 2012;3:61-72.

82. Government of Western Australia Department of Health. Guidelines for human biobanks, genetic research databases and associated data, 2010. Available from: http:/www.genomics.health.wa.gov.au/publications/ docs/guidelines_for_human_biobanks.pdf. Accessed May 7, 2014.

83. Eder J, Gottweis H, Zatloukal K. IT solutions for privacy protection in biobanking. Public Health Genomics. 2012;15:254-262.

84. Hansson MG, Gattorno M, Forsberg JS, Feltelius N, Martini A, Ruperto N. Ethics bureaucracy: a significant hurdle for collaborative follow-up of drug effectiveness in rare childhood diseases. Arch Dis Child. 2012;97:561-563. 
85. Hansson MG, van Ommen GJ, Chadwick R, Dillner J. Patients would benefit from simplified ethical review and consent procedure. Lancet Oncol. 2013;14:451-453.

86. Hallmans G, Vaught JB. Best practices for establishing a biobank. Methods Mol Biol. 2011;675:241-260.

87. Budin-Ljosne I, Harris JR, Kaye J, et al. ELSI challenges and strategies of national biobank infrastructures. Norsk Epidemiologi. 2012;21(2): 155-160.

88. Public Population Project in Genomics and Society. Charter of Principles. Available from: http://www.p3g.org/about-p3g/charterprinciples. Accessed February 11, 2014.

89. Steinsbekk KS, Kare Myskja B, Solberg B. Broad consent versus dynamic consent in biobank research: is passive participation an ethical problem? Eur J Hum Genet. 2013;21:897-902.
90. Stjernschantz Forsberg J, Hansson MG, Eriksson S. Biobank research: who benefits from individual consent? BMJ. 2011;343:5647.

91. Forsberg JS, Hansson MG, Eriksson S. The risks and benefits of re-consent. Science. 2011;332:306.

92. Hansson MG. Need for a wider view of autonomy in epidemiological research. BMJ. 2010;340:2335.

93. Hewitt R, Hainaut P. Biobanking in a fast moving world: an international perspective. J Natl Cancer Inst Monogr. 2011;2011:50-51.

94. Mascalzoni D, Dove ES, Rubinstein Y, et al. International Charter of principles for sharing bio-specimens and data. Eur J of Hum Genet. Epub 24 September, 2014

\section{Video abstract}

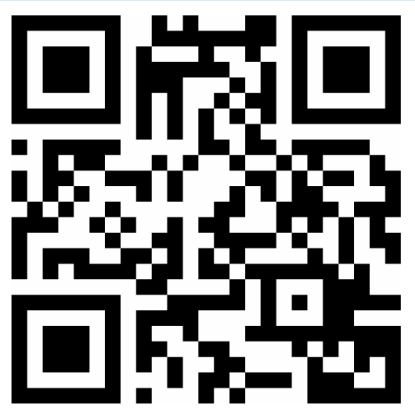

Point your SmartPhone at the code above. If you have a

QR code reader the video abstract will appear. Or use: http://dvpres/lyF2lo6
Journal of Biorepository Science for Applied Medicine

\section{Publish your work in this journal}

Journal of Biorepository Science for Applied Medicine is an international, peer-reviewed, open access journal that focuses on new developments and advances in the emergin and evolving field of biorepository science. This includes biospecimen procurement processing, preservation, and banking for application to applied medicine. The Journa invites submission of manuscripts which address these aspects in addition to system logic, clinical throughput and ethical issues pertaining to application of biorepositories

\section{Dovepress}

and their affects on clinical medicine. The journal is characterized by the rapid reporting of reviews, original research, methodologies, technologies and analytics in this subject area. The manuscript management system is completely online and includes a very quick and fair peer-review system, which is all easy to use. Visit http://www.dovepress. com/testimonials.php to read real quotes from published authors.

Submit your manuscript here: http://www.dovepress.com/journal-of-biorepository-science-for-applied-medicine-journal 